\title{
Randomised clinical studies investigating immediate and short-term efficacy of an occluding toothpaste in providing dentine hypersensitivity relief
}

Jonathan Creeth ${ }^{1 *} \mathbb{D}$, John Gallob², Farzana Sufi ${ }^{1}$, Jimmy Qaqish ${ }^{3}$, Paola Gomez-Pereira', Chandrashekhar Budhawant ${ }^{4}$ and Chhaju Goyal ${ }^{3}$

\begin{abstract}
Background: Dentine hypersensitivity $(\mathrm{DH})$ can occur after gum recession or enamel loss and may impact quality of life. Treatments include toothpastes that decrease DH by occluding dentine tubules. One effective occluding ingredient used in toothpastes is stannous fluoride $\left(\mathrm{SnF}_{2}\right)$, but this can be unstable in aqueous formulation. These three studies aimed to characterise the short-term effects of an experimental, anhydrous $\mathrm{SnF}_{2}$ dentifrice on $\mathrm{DH}$.

Methods: Three examiner-blind, parallel-group studies evaluated DH in participants with the condition after a single brushing and after $3 \mathrm{~d}$ brushing with an experimental anhydrous $0.454 \% \mathrm{SnF}_{2} /$ polyphosphate toothpaste (Test) or a toothpaste containing $\mathbf{0 . 7 6 \%}$ sodium monofluorophosphate (Control). Test treatment participants brushed two pre-identified sensitive teeth first, then their remaining dentition for $\geq 1$ min ('focused brushing'). Control treatment participants brushed their whole dentition for $\geq 1 \mathrm{~min}$. DH was measured after single brushing and after $3 d$ twice-daily use, via evaporative (air) (Schiff Sensitivity Scale) and tactile (Yeaple probe) stimuli and analysed using an ANCOVA model.
\end{abstract}

Results: In all studies, after $3 \mathrm{~d}$ treatment, the Test toothpaste/brushing regimen significantly reduced $\mathrm{DH}$ compared to the Control regimen by both evaporative and tactile stimuli assessment ( $p<0.0001$ for all). The Test regimen also significantly reduced DH from baseline at both time-points by both measures in all studies $(p<0.0001$ for all). Mean Schiff sensitivity score differences (95\% confidence intervals) between Test and Control regimens after 3d were: Study 1: -0.45 (-0.577, -0.319$)$; Study 2: $-0.40(-0.505,-0.300)$; Study 3: -1.31 ( $-1.500,-1.128)$. Mean tactile score differences were: Study 1: 11.30 (7.927, 14.662); Study 2: 3.57 (2.531, 4.614); Study 3: 24.54 (20.349, 28.736). After single use, in Studies 2 and 3, the Test toothpaste/brushing regimen significantly reduced DH versus Control by both measures ( $p<0.001$ for all); in Study 1, treatment differences were not significant. Toothpastes were generally well-tolerated.

Conclusions: Taken together, these studies indicated focused brushing with an experimental anhydrous $0.454 \%$ $\mathrm{SnF}_{2}$ /polyphosphate toothpaste reduces $\mathrm{DH}$ compared to brushing with a conventional toothpaste after single use, with greater reduction after $3 \mathrm{~d}$.

Trial registration: Registrations at ClinicalTrials.gov: Study 1: NCT02832375 (registered 26.July.2016); Study 2: NCT02731833 (registered 26.April.2016); Study 3: NCT02923895 (registered 5.October.2016).

Keywords: Dentin hypersensitivity, Tin fluorides, Clinical trial, Toothpastes

\footnotetext{
* Correspondence: jonathan.e.creeth@gsk.com

'GSK Consumer Healthcare, Weybridge, Surrey, UK

Full list of author information is available at the end of the article
}

(c) The Author(s). 2019 Open Access This article is distributed under the terms of the Creative Commons Attribution 4.0 International License (http://creativecommons.org/licenses/by/4.0/), which permits unrestricted use, distribution, and reproduction in any medium, provided you give appropriate credit to the original author(s) and the source, provide a link to the Creative Commons license, and indicate if changes were made. The Creative Commons Public Domain Dedication waiver (http://creativecommons.org/publicdomain/zero/1.0/) applies to the data made available in this article, unless otherwise stated. 


\section{Background}

Gum recession or enamel loss can lead to the underlying dentine becoming exposed $[1,2]$. A thermal, tactile, chemical, osmotic or evaporative stimulus, such as a hot drink or cold air, is believed to lead to movement of the fluid contained within dentine tubules, which in turn stimulates pupal nerve fibres and results in a short, sharp pain characteristic of dentine hypersensitivity (DH), as detailed in the 'hydrodynamic theory' of DH [3-5]. DH has been shown to have a negative impact on people's oral health-related quality of life [6], affecting such aspects as eating, drinking, talking and participating in outdoor activities [7].

There are two predominant methods to treat $\mathrm{DH}$ using oral care products: nerve desensitisation and tubule occlusion. Potassium ions (a nerve desensitiser) $[8,9]$ may need repeated administration over a number of weeks before symptomatic relief is achieved [10]. The latter method, occlusion of dentine tubules, relies on ingredients such as strontium or stannous salts $[8,11,12]$, arginine-calcium carbonate $[9,13]$ or bioglasses $[14,15]$. These form solid deposits in the exposed ends of dentine tubules that physically block them so an external stimulus does not reach the fluid held within [8-10]. This approach has the potential to work more rapidly than desensitisation.

Previous studies of anti-sensitivity formulations containing occluding agents have found advantages for shortterm relief of DH when the toothpaste is applied in a focused manner to the affected sensitive teeth. This may be achieved by massaging a small amount of toothpaste into each sensitive area via a finger-tip (the 'dab-on' technique) $[12,16-20]$ or by 'focused brushing' of the affected teeth, where the sensitive areas are brushed with toothpaste before the rest of the dentition [21-24]. The 'focused brushing' technique is of interest as it is easily incorporated into a normal toothbrushing routine.

Stannous fluoride $\left(\mathrm{SnF}_{2}\right)$ is a particularly interesting option as an occluding agent as it provides both the potential for sensitivity relief and a source of free fluoride ions for protection against dental caries. It is also compatible with most other conventional toothpaste ingredients and is relatively taste-neutral. The stannous ion is the active component which rapidly oxidises (from $\mathrm{Sn}[\mathrm{II}]$ to $\mathrm{Sn}[\mathrm{IV}])$ and hydrolyses in the presence of saliva to form insoluble tin compounds (hydroxides, oxides and phosphates) [25]. Stannous ions have been shown in vitro to form insoluble precipitates on the dentine surface that occlude dentine tubules through combination with formulation excipients and saliva-derived ions [25, 26].

$\mathrm{SnF}_{2}$ has been used in toothpastes to relieve $\mathrm{DH}$ for many decades [27] and has shown evidence of clinical efficacy in both short-term and long-term studies [12-24, $27-30]$. Since the discovery of the anti-DH properties of $\mathrm{SnF}_{2}$, toothpaste formulation development in the industry has concentrated on maximising stability (avoiding premature oxidation and hydrolysis) and delivery to the site of action, while minimising the staining effects of stannous ions [31, 32]. Use of anhydrous formulations of $\mathrm{SnF}_{2}$ leads to products that are stable on storage but readily hydrate and become active on exposure to saliva and the aqueous oral environment [33-35]. The addition of polyphosphates can control stannous ion-induced dental stain build-up [36]. This $\mathrm{SnF}_{2}$ with polyphosphate anhydrous formulation approach has been shown to occlude dentine tubules in vitro [33, 37, 38], with clinical studies over $8 \mathrm{wk}$. showing a reduction in DH $[24,27]$ and limitation of dental stain build-up [36]. However, not all published data for $\mathrm{DH}$ relief after just a single focused brushing is positive [29].

Further development of the anhydrous $\mathrm{SnF}_{2}$ formulation has since been completed by GSK Consumer Healthcare. This work aimed to improve the ability of the formulation to deliver stannous ions rapidly to the site of action at the dentine surface and retain it there by optimising the polymer thickening system. The resulting new experimental formulation has been shown in vitro to increase the rate of tubule occlusion compared to an existing anhydrous $\mathrm{SnF}_{2}$ / polyphosphate formulation (unpublished findings).

Following these in vitro results, clinical studies were needed to investigate the effects of the new experimental formulation on DH. Three such studies were undertaken, which are the subject of this report. The aim of these three studies was to evaluate the ability of an experimental $0.454 \% \mathrm{SnF}_{2}$ /sodium tripolyphosphate (STP) anhydrous toothpaste to provide relief from $\mathrm{DH}$ when applied by the 'focused brushing' technique prior to whole mouth brushing, as elicited by evaporative (air) and tactile stimuli after $3 \mathrm{~d}$ twice-daily use and after a single application, compared to a regular toothpaste containing $0.76 \%$ sodium monofluorophosphate (SMFP) applied by conventional toothbrushing.

\section{Methods}

These three studies closely followed the protocol described in a previous investigation by some of the current authors (Gallob et al. [39]). The studies used a $3 \mathrm{~d}$, randomised, examiner-blind, two treatment-arm, parallel design. In accordance with consensus guidelines [40], two independent, stimulus-based clinical measures were used to assess DH: evaporative (air) sensitivity (Schiff Sensitivity score [41]) and sensitivity to tactile stimulus (via a constant-pressure Yeaple probe [42]). Study 1 was conducted by Silverstone Research Group, USA; Studies 2 and 3 were conducted by All Sum Research Centre, Canada (on separate populations). All studies were conducted in accordance with the Declaration of Helsinki and approved by independent institutional review boards before initiation (Study 1: US Institutional Review Board, Inc., Miami, FL, 
USA, Reference number: U.S.IRB2016SRG/01; Study 2: Veritas IRB, Montreal, Canada, Reference number: 1604516-02:5011-03-2016; Study 3: Veritas IRB, Montreal, Canada Reference number: 16087-12:05:1921-12-2016). These studies are registered at ClinicalTrials.gov: Study 1: NCT02832375; Study 2: NCT02731833; Study 3: NCT02923895.

\section{Participants}

Criteria for eligibility for the studies were as used in the previous investigation [39], modified as described below.

Inclusion criteria: participants needed to be $18-65 \mathrm{yr}$. with a self-reported history of DH lasting between $6 \mathrm{mth}$ and $10 \mathrm{yr}$., with at least two accessible teeth (incisors, canines or premolars, non-adjacent) with a positive response to an evaporative air stimulus [41], that had a Modified Gingival Index (MGI) [43] score of 0, signs of facial/cervical erosion, abrasion and/or gingival recession $($ EAR) and a clinical mobility score $\leq 1$ [44]

Exclusion criteria: participants in Study 2 could not participate in Study 3; professional desensitising treatment within 8 wk. of Screening was not a stated exclusion criterion, other than for test teeth in study 3; and certain general dental exclusions in the previous study applied to test teeth only in this case (exposed dentine with deep, defective or facial restorations; teeth used as abutments for fixed/removable partial dentures; full crowns or veneers; orthodontic bands; cracked enamel and sensitive teeth with contributing aetiologies other than EAR; presence of dental implants in Study 3 only).

\section{Procedures}

Procedures at the screening visit followed those of Gallob et al. [39]; dentition was assessed for eligibility as described above.

Eligible participants were supplied with a conventional fluoride toothpaste (Crest Cavity Protection; Procter \& Gamble, Cincinnati, OH; USA; USA/Canadian marketed product; $1000 \mathrm{ppm}$ fluoride as sodium fluoride) and a toothbrush (Studies 1 and 2: Aquafresh Clean Control [Everyday Clean], GSK Consumer Healthcare, Weybridge, UK [GSKCH]; Study 3: Oral-B Sensi-soft Manual Toothbrush; Proctor \& Gamble, Cincinnati, OH, USA). Following first use under study site supervision, participants used this toothpaste twice daily during the acclimatisation period between screening and baseline visits: 4-6 wk. (Studies 1 and 2), 4-8 wk. (Study 3).

Procedures for the Baseline and $3 \mathrm{~d}$ visits also followed those of Gallob et al. [39], other than: participants refrained from taking analgesics for at least $8 \mathrm{~h}$ prior to the study visit, and subjects were not permitted to chew gum during the study. In each study, a single examiner performed all assessments for the duration of the trial.
Eligible participants were assigned randomly to one of two toothpaste/brushing regimens: one using the 'Test' toothpaste, containing $0.454 \% \mathrm{SnF}_{2}$ (1100 ppm fluoride) and 5\% STP, the other using the 'Control' toothpaste, containing $0.76 \%$ SMFP (1000 ppm fluoride) (Colgate Cavity Protection; Colgate-Palmolive, New York, NY, USA; USA marketed product).

Subjects were stratified according to their maximum baseline Schiff sensitivity score of the two selected teeth, to ensure balance between the Test and Control regimen groups. Subjects with maximum baseline Schiff sensitivity score of 2 for the two selected test teeth were allocated to Stratum 1; those with maximum score of 3 were allocated to Stratum 2. Randomisation numbers within each stratum were assigned in increasing numerical order, according to appearance at the study site on the day subjects were randomised, once eligibility was determined. Assignment was performed according to a randomisation schedule provided by the Biostatistics department of the study sponsor.

Toothpaste/brushing regimen allocation was kept blind to the dental examiner, study statistician, data management staff and other employees of the sponsor who could have influenced study outcomes. To help blind participants to product identity, study product tubes were overwrapped with white vinyl.

Clinical assessments of DH to evaporative (air) and tactile stimuli were made after the first use of the assigned toothpaste, at the study site. All participants brushed with a full ribbon of toothpaste. In Studies 1 and 2, participants in the Test treatment group were instructed to brush each of the two test teeth first, then the whole dentition for at least $1 \mathrm{~min}$. The duration of focused brushing was not specified in studies 1 and 2; in Study 3 (which occurred after studies 1 and 2), it was set at $30 \mathrm{~s}$ per tooth, to standardise product use [21-23]. Control group participants in all studies were instructed to brush the whole dentition for at least $1 \mathrm{~min}$. All participants were permitted to rinse with tap water following brushing.

Participants then brushed twice a day (morning and evening) in this manner for $3 \mathrm{~d}$, following which $\mathrm{DH}$ assessments were repeated. Compliance with use of the study toothpaste was assessed by review of participantcompleted diary cards.

\section{Safety}

Adverse events (AEs) and any abnormalities in the OST examination were recorded from the start of use with the acclimatisation toothpaste at the screening visit until $5 \mathrm{~d}$ after the last use of study toothpaste. The investigator assessed whether an $\mathrm{AE}$ was treatment-related or not and graded it as mild, moderate or severe. 


\section{Data analysis}

Based on outcomes from previous sensitivity studies (unpublished findings), for Study 1, it was planned to screen sufficient participants to enter approximately 250 into the acclimatisation phase so as to randomise approximately 240 and ensure approximately 214 participants completed the study. For Study 2, participant numbers were: acclimatisation 240; randomisation 235; for 214 to complete. For Study 3, participant numbers were: acclimatisation 210; randomisation 190; for 184 to complete (in which the sample size was also based on the results of Studies 1 and 2, which preceded it, and unpublished findings).

The primary endpoint was change from baseline (mean of the two selected test teeth) in evaporative (air) sensitivity after $3 \mathrm{~d}$, as recorded on the Schiff Sensitivity Scale. Secondary endpoints were: change from baseline in Schiff sensitivity score after a single application; change in tactile threshold after a single application; and change in tactile threshold after $3 \mathrm{~d}$. The primary objective was to determine whether there was a difference in primary endpoint between the Test and Control toothpaste/brushing regimens, the null hypothesis being that there was no difference.

For Studies 1 and 2, it was estimated that using 107 participants per group would have an $80 \%$ power to detect a mean difference between treatments of 0.25 units in Schiff sensitivity score (assuming a standard deviation [SD] of 0.6487 ) using a two-sided t-test of significance level 0.05 . This represents a clinically significant difference after $3 \mathrm{~d}$ of treatment. For Study 3, it was estimated that a sample of 92 participants per group would have a $90 \%$ power to detect a mean difference between the treatments of 0.25 units in Schiff sensitivity score (assuming a SD of 0.5198) using the same significance level.

Efficacy analyses were performed on the intent-to-treat (ITT) population, defined as all randomised participants who provided at least one post-baseline assessment of efficacy. The per-protocol (PP) population was defined as all participants in the ITT population who had at least one efficacy assessment unaffected by protocol violations.

Change from baseline was evaluated by analysis of covariance (ANCOVA), with treatment group as a factor and the corresponding baseline score (Schiff sensitivity score or tactile threshold) as a covariate. For tactile threshold, the maximum baseline Schiff sensitivity score stratum of the two selected test teeth was included as a factor. For all treatment groups, baseline means, adjusted means for the between-treatment differences, 95\% confidence intervals (CIs) and $p$-values for treatment comparisons are presented. All tests were conducted at the two-sided 5\% significance level, with no adjustments for multiple testing as the primary comparison was pre-defined.

\section{Results}

The ANCOVA model assumptions for the analyses of Schiff sensitivity score were investigated and considered to be satisfied for all studies. For the tactile threshold data, there was some evidence of departure from the model assumptions, therefore change in tactile threshold was also analysed by a non-parametric method (van Elteren test, adjusting for the maximum baseline Schiff sensitivity score) and the results compared with the ANCOVA results. The inferences from the two analyses were similar, thus emphasis has been given to the ANCOVA results.

\section{Participants: Tables 1 and 2.}

For Study 1, 242 participants were randomised to treatment. The first participant was enrolled on 28 March 2016, the last completed the study on 12 May 2016. Of the 242 participants in the safety population, the majority were female $(n=154 ; 63.6 \%)$ and were in Schiff stratum ' 3 ' ( $n=177 ; 73.1 \%)$; the mean age was $37.7 \mathrm{yr}$. (SD: 11.15; range 20-65 yr).

For Study 2, 222 participants were randomised to treatment. The first participant was enrolled on 4 April 2016, the last completed the study on 20 May 2016. Of the 222 participants in the safety population, the majority were female $(n=163 ; 73.4 \%)$ and were in Schiff stratum ' 3 ' ( $n=119 ; 53.6 \%)$; the mean age was $47.0 \mathrm{yr}$. (SD: 10.85 ; range $18-65 \mathrm{yr}$ ).

For Study 3, 192 participants were randomised to treatment. The first participant was enrolled on 11 October 2016, the last completed the study on 16 December 2016. Of the 192 participants in the safety population, the

Table 1 Participant disposition through study

\begin{tabular}{|c|c|c|c|c|c|c|}
\hline & \multicolumn{6}{|c|}{ Number of participants } \\
\hline & \multicolumn{2}{|c|}{ Study 1} & \multicolumn{2}{|c|}{ Study 2} & \multicolumn{2}{|c|}{ Study 3} \\
\hline \multicolumn{7}{|l|}{ Enrolment } \\
\hline Total screened & \multicolumn{2}{|l|}{266} & \multicolumn{2}{|l|}{229} & \multicolumn{2}{|l|}{197} \\
\hline Randomised & \multicolumn{2}{|l|}{242} & \multicolumn{2}{|l|}{222} & \multicolumn{2}{|l|}{192} \\
\hline Not randomised & \multicolumn{2}{|l|}{24} & \multicolumn{2}{|l|}{7} & \multicolumn{2}{|l|}{5} \\
\hline Not eligible & \multicolumn{2}{|l|}{14} & \multicolumn{2}{|l|}{7} & \multicolumn{2}{|l|}{1} \\
\hline Withdrew consent & \multicolumn{2}{|l|}{9} & \multicolumn{2}{|l|}{0} & \multicolumn{2}{|l|}{4} \\
\hline Lost to follow-up & \multicolumn{2}{|l|}{1} & \multicolumn{2}{|l|}{0} & \multicolumn{2}{|l|}{0} \\
\hline Allocation & Test & Control & Test & Control & Test & Control \\
\hline Randomised/received & 121 & 121 & 111 & 111 & 97 & 95 \\
\hline \multicolumn{7}{|l|}{ Follow-up } \\
\hline Completed study & 119 & 121 & 111 & 111 & 97 & 95 \\
\hline Withdrew consent & 1 & 0 & 0 & 0 & 0 & 0 \\
\hline Lost to follow-up & 1 & 0 & 0 & 0 & 0 & 0 \\
\hline \multicolumn{7}{|l|}{ Analysis } \\
\hline Safety/ITT/PP populations & 121 & 121 & 111 & 111 & 97 & 95 \\
\hline
\end{tabular}


Table 2 Summary of demographic and baseline characteristics (safety population)

\begin{tabular}{|c|c|c|c|c|c|c|}
\hline \multirow[t]{2}{*}{ Characteristic } & \multicolumn{2}{|l|}{ Study 1} & \multicolumn{2}{|l|}{ Study 2} & \multicolumn{2}{|l|}{ Study 3} \\
\hline & Test $(n=121)$ & Control $(n=121)$ & Test $(n=111)$ & Control $(n=111)$ & Test $(n=97)$ & Control $(n=95)$ \\
\hline \multicolumn{7}{|l|}{ Sex, n (\%) } \\
\hline Male & $45(37.2)$ & $43(35.5)$ & $29(26.1)$ & $30(27.0)$ & $29(29.9)$ & $23(24.2)$ \\
\hline Female & $76(62.8)$ & $78(64.5)$ & $82(73.9)$ & $81(73.0)$ & $68(70.1)$ & $72(75.8)$ \\
\hline \multicolumn{7}{|l|}{ Age, yr } \\
\hline Mean (SD) & $38.0(11.32)$ & $37.4(11.01)$ & $46.4(10.77)$ & $47.6(10.95)$ & $46.8(12.95)$ & $46.9(10.53)$ \\
\hline Range & $20-66$ & $20-64$ & $19-64$ & $18-65$ & $19-65$ & $19-65$ \\
\hline \multicolumn{7}{|l|}{ Race, n (\%) } \\
\hline White & $74(61.2)$ & $72(59.5)$ & $60(54.1)$ & $73(65.8)$ & $62(63.9)$ & $51(53.7)$ \\
\hline Black/African American & $25(20.7)$ & $27(22.3)$ & $33(29.7)$ & $23(20.7)$ & $18(18.6)$ & $31(32.6)$ \\
\hline Asian & $9(7.4)$ & $10(8.3)$ & $18(16.2)$ & $15(13.5)$ & $16(16.5)$ & $12(12.6)$ \\
\hline Other & $13(10.7)$ & $12(9.9)$ & 0 & 0 & $1(1.0)$ & $1(1.1)$ \\
\hline Schiff stratum 2, n (\%) & $33(27.3)$ & $32(26.4)$ & $52(46.8)$ & $51(45.9)$ & $45(46.4)$ & $43(45.3)$ \\
\hline Schiff stratum 3, n (\%) & $88(72.7)$ & $89(73.6)$ & $59(53.2)$ & $60(54.1)$ & $52(53.6)$ & $52(54.7)$ \\
\hline
\end{tabular}

majority were female $(n=140 ; 72.9 \%)$ and were in Schiff stratum ' 3 ' ( $n=104 ; 54.2 \%)$; the mean age was 46.9 yr. (SD: 11.79; range 19-65 yr).

The Baseline characteristics of the treatment groups were similar between groups for the safety and ITT populations of each study: in none of the studies was there a difference between the ITT and Per-Protocol populations.

\section{Efficacy}

In all studies, the Test toothpaste/brushing regimen showed a statistically significant decrease from baseline in Schiff sensitivity scores (Fig. 1) and increase from baseline in tactile threshold (Fig. 2) after both single and $3 \mathrm{~d}$ use $(p<0.0001$ for all) (Table 3). In Study 1 only, the
Control toothpaste/brushing regimen showed a statistically significant reduction in $\mathrm{DH}$ from baseline, as detected via both measures at both timepoints $(p<0.002$ for all).

In all studies, these changes from baseline were significantly greater after $3 \mathrm{~d}$ for the Test product than the Control. Test versus Control differences ranged across the three studies from 0.40 to 1.31 units in Schiff sensitivity score, and from $3.57 \mathrm{~g}$ to $24.54 \mathrm{~g}$ in tactile threshold (Table 3). Furthermore, in Studies 2 and 3, the reduction in $\mathrm{DH}$ was significantly greater for the Test product than for the Control after a single use. The differences were greatest in Study 3; all favoured the Test dentifrice.

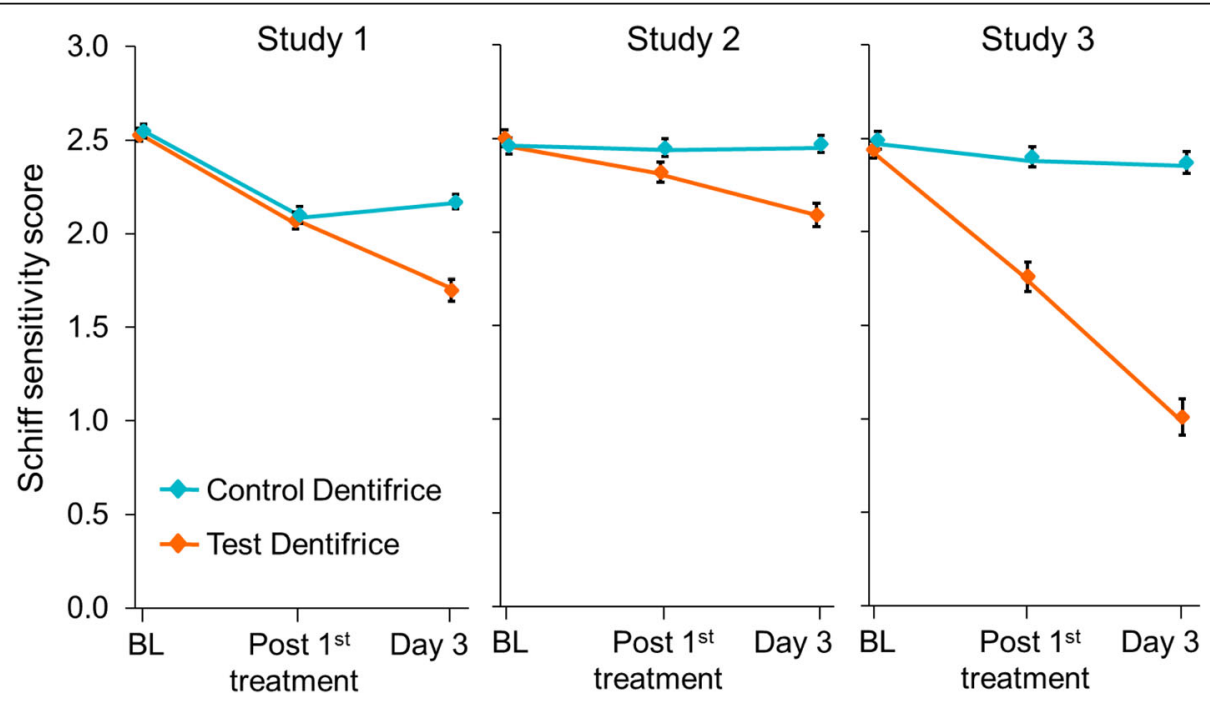

Fig. 1 Mean ( \pm standard error) Schiff sensitivity scores (intent-to-treat population). Data are offset for clarity. BL: Baseline 


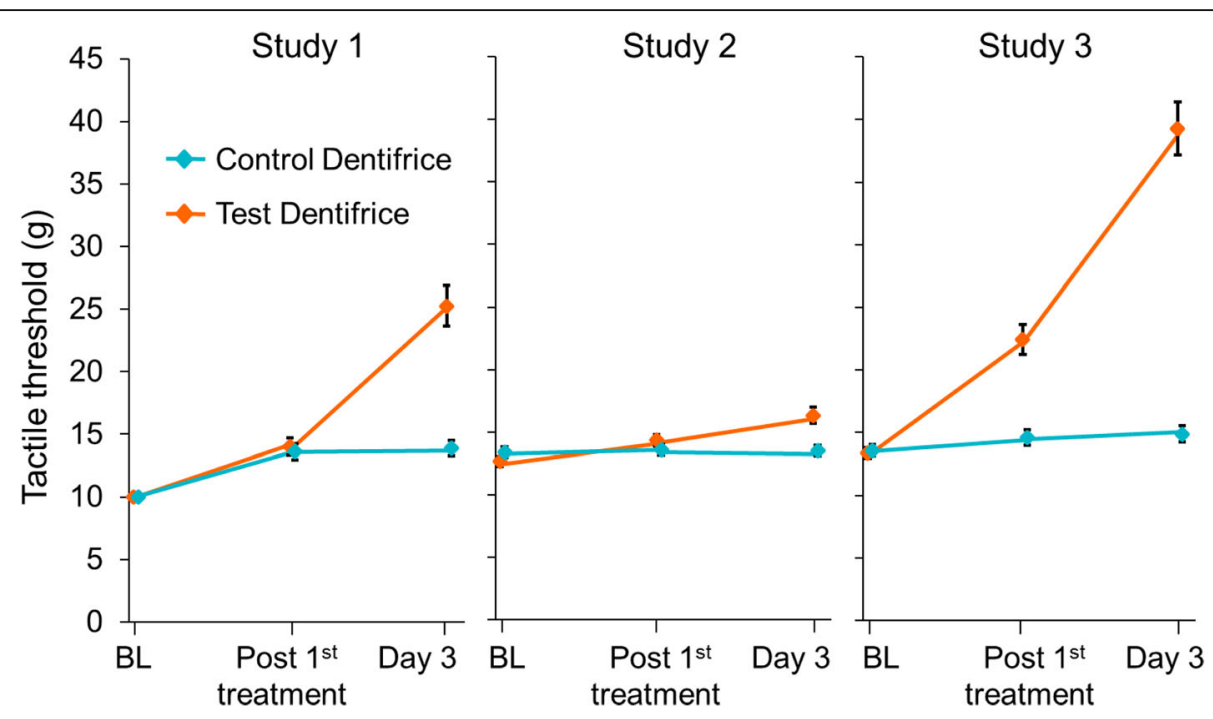

Fig. 2 Mean ( \pm standard error) tactile threshold scores (intent-to-treat population). Data are offset for clarity. BL: Baseline

Table 3 Statistical analysis of change from baseline in Schiff sensitivity score and tactile threshold (intent-to-treat population)

\begin{tabular}{|c|c|c|c|c|}
\hline & & Test* & Control* $^{*}$ & Test vs Cont ${ }^{* *}$ \\
\hline \multicolumn{5}{|c|}{ Schiff sensitivity score } \\
\hline \multirow[t]{3}{*}{ Study 1} & $B L$ & $2.53(0.035)$ & $2.55(0.036)$ & \\
\hline & $0 d$ & $-0.46(-0.55,-0.38) \boldsymbol{p}<\mathbf{0 . 0 0 0 1}$ & $-0.45(-0.53,-0.37) \boldsymbol{p}<\mathbf{0 . 0 0 0 1}$ & $-0.01(-0.131,0.102) p=0.8067$ \\
\hline & $3 d$ & $-0.83(-0.92,-0.74) \boldsymbol{p}<\mathbf{0 . 0 0 0 1}$ & $-0.38(-0.47,-0.29) \boldsymbol{p}<\mathbf{0 . 0 0 0 1}$ & $-0.45(-0.577,-0.319) \boldsymbol{p}<\mathbf{0 . 0 0 0 1}$ \\
\hline \multirow[t]{3}{*}{ Study 2} & $B L$ & $2.5(0.046)$ & $2.46(0.044)$ & \\
\hline & $0 d$ & $-0.17(-0.23,-0.11) \boldsymbol{p}<\mathbf{0 . 0 0 0 1}$ & $-0.02(-0.08,0.04) p=0.5089$ & $-0.15(-0.232,-0.070) \boldsymbol{p}=\mathbf{0 . 0 0 0 3}$ \\
\hline & $3 d$ & $-0.40(-0.47,-0.33) \boldsymbol{p}<\mathbf{0 . 0 0 0 1}$ & $0.00(-0.07,0.08) p=0.9298$ & $-0.40(-0.505,-0.300) \boldsymbol{p}<\mathbf{0 . 0 0 0 1}$ \\
\hline \multirow[t]{3}{*}{ Study 3} & $B L$ & $2.44(0.046)$ & $2.49(0.049)$ & \\
\hline & $0 d$ & $-0.69(-0.80,-0.57) \boldsymbol{p}<\mathbf{0 . 0 0 0 1}$ & $-0.09(-0.20,0.03) p=0.1281$ & $-0.60(-0.759,-0.435) \boldsymbol{p}<\mathbf{0 . 0 0 0 1}$ \\
\hline & $3 d$ & $-1.44(-1.57,-1.31) \boldsymbol{p}<\mathbf{0 . 0 0 0 1}$ & $-0.13(-0.26,0.01) p=0.0630$ & $-1.31(-1.500,-1.128) \boldsymbol{p}<\mathbf{0 . 0 0 0 1}$ \\
\hline \multicolumn{5}{|c|}{ Tactile threshold } \\
\hline \multirow[t]{3}{*}{ Study 1} & $B L$ & $10.00(0.000)$ & $10.00(0.000)$ & \\
\hline & $0 d$ & $+4.00(2.68,5.31) \boldsymbol{p}<\mathbf{0 . 0 0 0 1}$ & $+3.60(2.29,4.92) \boldsymbol{p}<\mathbf{0 . 0 0 0 1}$ & $0.39(-1.465,2.253) p=0.6770$ \\
\hline & $3 d$ & $+15.22(12.83,17.61) \boldsymbol{p}<\mathbf{0 . 0 0 0 1}$ & $+3.92(1.55,6.29) \boldsymbol{p}=\mathbf{0 . 0 0 1 3}$ & $11.30(7.937,14.662) \boldsymbol{p}<0.0001$ \\
\hline \multirow[t]{3}{*}{ Study 2} & $B L$ & $12.66(0.399)$ & $13.42(0.405)$ & \\
\hline & $0 d$ & $+1.67(1.14,2.19) \boldsymbol{p}<\mathbf{0 . 0 0 0 1}$ & $+0.23(-0.30,0.75) p=0.4001$ & $1.44(0.694,2.188) \boldsymbol{p}=\mathbf{0 . 0 0 0 2}$ \\
\hline & $3 d$ & $+3.68(2.94,4.41) \boldsymbol{p}<\mathbf{0 . 0 0 0 1}$ & $+0.11(-0.63,0.84) p=0.7775$ & $3.57(2.531,4.614) \boldsymbol{p}<\mathbf{0 . 0 0 0 1}$ \\
\hline \multirow[t]{3}{*}{ Study 3} & $B L$ & $13.40(0.431)$ & $13.58(0.453)$ & \\
\hline & $0 d$ & $+9.05(7.25,10.85) \boldsymbol{p}<\mathbf{0 . 0 0 0 1}$ & $+1.07(-0.75,2.89) p=0.2461$ & $7.98(5.423,10.539) \boldsymbol{p}<0.0001$ \\
\hline & $3 d$ & $+25.87(22.92,28.82) \boldsymbol{p}<\mathbf{0 . 0 0 0 1}$ & $+1.32(-1.66,4.30) p=0.3816$ & $24.54(20.349,28.736) \boldsymbol{p}<\mathbf{0 . 0 0 0 1}$ \\
\hline
\end{tabular}

Baseline values are raw means (standard error), and only include participants with a corresponding post-baseline assessment, for post-baseline visit output is obtained from ANCOVA model

*Change from baseline $(95 \% \mathrm{Cl}) \mathrm{p}$-value

**Difference $(95 \% \mathrm{Cl})$ p-value: first-named minus second-named group such that, for Schiff sensitivity score, a negative difference favours the first-named group or, for tactile threshold, a positive difference favours the first-named group

BL: Baseline; d: day

$P$-values in bold are below the $p=0.05$ threshold 


\section{Safety}

In Study 1 there were three reported TEAEs, by one participant in the Test group (oral mucosal exfoliation) and two in the Control group (oral leucoplakia and lip ulceration). Of these, only oral mucosal exfoliation was considered treatment-related. There were no TEAEs in Study 2. In Study 3 there was one TEAE in the Test group (headache), that was not considered treatment-related. In all studies, all TEAEs were mild and had resolved by study end. There were no withdrawals in any of the studies due to a TEAE. No serious adverse events or incidents were reported.

\section{Discussion}

Previous short-term studies have found advantages in DH relief when a toothpaste is applied in a focused manner to the affected sensitive teeth, a routine easily incorporated into normal toothbrushing. However, for $\mathrm{SnF}_{2}$, not all clinical studies have shown DH relief after just a single application by focused brushing [29]. In this report, a series of three studies showed that an experimental toothpaste containing $0.454 \% \mathrm{SnF}_{2} / 5 \% \mathrm{STP}$ applied by focused brushing significantly improved $\mathrm{DH}$ using two separate measures, after a single application (in two of the studies) and $3 \mathrm{~d}$ use, compared to conventional brushing with a regular toothpaste. The primary endpoint, of a difference in Schiff sensitivity score between the Test toothpaste/brushing regimen and the Control after $3 \mathrm{~d}$ use, was met in all studies. Across the three trials, the range in mean change in Schiff score (0.40-1.31 Schiff units) indicated there was a clinically meaningful difference between the effects of the Test and Control product toothpaste/brushing regimens [45]. The differences observed using the tactile stimulus paralleled these findings. These results compare well with previous studies employing this technique for stannous fluoride dentifrices, where effect sizes up to about 1.2 Schiff units have been reported after 3 days use [21-23].

Overall, a reduction in DH due to the Test toothpaste/ brushing regimen was detected in the three studies reported here; however, differences in degree of effect were observed (Figs. 1 and 2/Table 3). This may be explained by the fact that any single study will only estimate the true difference between treatments; normal biological variation will mean some studies will over-estimate the true difference and some under-estimate. In this set of studies, after a single application, a statistically significant between-treatment difference was shown in Studies 2 and 3 for both sensitivity measures; in Study 1, however, there were no significant between-treatment changes on either measure. Only in this latter study did the Control toothpaste/brushing regimen show significant changes from baseline with both Schiff and tactile stimuli after a single application: it has been noted that both placebo and
Hawthorne effects can affect DH studies and these may have occurred here [46-48]. It is also a possibility that there were more cases of $\mathrm{DH}$ severity reducing without treatment during Study 1 than in the other studies, suggested by the high proportion of Schiff score 3 at Baseline [46-48].

A 'focused brushing' technique was used by participants in the Test treatment group; those in the Control group brushed without specifically treating sensitive areas. This approach was taken to follow that of He et al. [21-23], aiming to mimic a real-world situation in which people with DH using either product would follow the brushing instructions for that toothpaste. Study 3 standardised the application time of the Test treatment during the focused brushing element of the brushing routine to $30 \mathrm{~s}$, prior to whole mouth brushing. This may have contributed to the greater difference between Test and Control treatments observed, but many other factors including participant population, and time of year, also differed.

Despite the small differences observed between the studies, their results provide further evidence that $\mathrm{SnF}_{2}$ delivered by focused and whole mouth brushing with a toothpaste can be an effective tubule occluding agent, in line with in vitro studies $[33,37,40]$. The mechanism of action of the optimised polymer system of this formulation is postulated to be to promote stannous ion deposition and retention (as various tin salts). In vitro hydraulic conductance studies found that application of the Test toothpaste lowered fluid flow rate after both a single treatment and after three treatments over $48 \mathrm{~h}$, as well as following a challenge with an acidic drink; these were statistically significantly greater than a similar commercial formulation containing $\mathrm{SnF}_{2}$ (unpublished findings). Moreover, fluid flow rate decreased with each subsequent treatment, suggesting cumulative effects of daily application. This is consistent with the observations in the three clinical studies presented here, in which beneficial changes in Schiff sensitivity score and tactile threshold recorded after single use were observed to increase after $3 \mathrm{~d}$ of consecutive use.

\section{Conclusions}

The present study set shows that an experimental anhydrous toothpaste formulation, containing $0.454 \%$ $\mathrm{SnF}_{2}$ with an optimised polymer system, can reduce DH when applied using the focused brushing technique, relative to conventional brushing with a regular fluoride toothpaste, after only a single use. This reduction in DH builds with subsequent twice-daily applications over the ensuing $3 \mathrm{~d}$. The short-term, cumulative nature of this benefit should meaningfully improve oral health-related quality of life for people with dentine hypersensitivity. 


\section{Abbreviations}

AE: Adverse event; ANCOVA: Analysis of covariance; BL: Baseline; Cl: Confidence intervals; DH: Dentine hypersensitivity; EAR: Erosion, abrasion and/or gingival recession; ITT: Intent-to-treat; MGI: Modified Gingival Index; OST: Oral soft tissue; PP: Per-protocol; SD: Standard deviation; SMFP: Sodium monofluorophosphate; Sn[II]: Tin [II]; Sn[IV]: Tin [IV]; SnF : Stannous fluoride; STP: Sodium tripolyphosphate

\section{Acknowledgements}

The authors would like to thank study staff and participants as well as Nisha Patel for help with study design and interpretation, Nan Wang and Darren Targett, both formerly of GSK Consumer Healthcare, for statistical analysis and Eleanor Roberts of Beeline Science Communications, Ltd., and Claire Hall of Raven Lane Consulting for help with manuscript preparation.

\section{Funding}

This study was funded by GSK Consumer Healthcare. Between them, the GSK-employed authors (JC, FS and PGP) contributed to all aspects of the study: design, data collection and analysis, interpretation of data and preparation of the manuscript.

\section{Availability of data and materials}

Anonymized individual participant data and study documents can be requested for further research from www.clinicalstudydatarequest.com.

\section{Authors' contributions}

$J C, J G, C G, F S$ and PGP developed the study protocol. JG, JQ and CG were involved in data acquisition. JC, FS, PGP and CB were involved in data analysis and interpretation. All authors contributed to the drafting of the manuscript and approved the final copy.

\section{Ethics approval and consent to participate}

Each participant provided written informed consent to participate in the study. All studies were conducted in accordance with the Declaration of Helsinki and approved by independent institutional review boards before initiation (Study 1: US Institutional Review Board, Inc., Miami, FL, USA, Reference number: U.S.IRB2016SRG/01; Study 2: Veritas IRB, Montreal, Canada, Reference number: 16045-16-02:5011-03-2016; Study 3: Veritas IRB, Montreal, Canada Reference number: 16087-12:05:1921-12-2016). These studies are registered at ClinicalTrials.gov: Study 1: NCT02832375; Study 2: NCT02731833; Study 3: NCT02923895.

\section{Consent for publication}

N/A: no details, images, or videos relating to an individual person are included in the manuscript.

\section{Competing interests}

Jonathan Creeth, Farzana Sufi and Paola Gomez Pereira are employees of GSK Consumer Healthcare, who funded the study and developed the Test product that was the subject of this manuscript.

Silverstone Research, of whom John Gallob is an employee, All Sum Clinical Research, of whom Chhaju Goyal and Jimmy Qaqish are employees, and Syneos Health, of whom Chandrashekhar Budhawant is was an employee at the time of the study, have all received funding from GSK Consumer Healthcare.

\section{Publisher's Note}

Springer Nature remains neutral with regard to jurisdictional claims in published maps and institutional affiliations.

\section{Author details}

${ }^{1}$ GSK Consumer Healthcare, Weybridge, Surrey, UK. ${ }^{2}$ Silverstone Research, Las Vegas, NV, USA. ${ }^{3}$ All Sum Clinical Research, Mississauga, Ontario, Canada. ${ }^{4}$ Syneos Health Clinical, Hyderabad, India.
Received: 31 July 2018 Accepted: 8 May 2019

Published online: 04 June 2019

\section{References}

1. Rimondini L, Baroni C, Carrassi A. Ultrastructure of hypersensitive and non-sensitive dentine. A study on replica models. J Clin Periodontol. 1995:22:899-902.

2. West NX, Lussi A, Seong J, Hellwig E. Dentin hypersensitivity: pain mechanisms and aetiology of exposed cervical dentin. Clin Oral Investig. 2013;17(Suppl 1):95-195.

3. Brännström M. A hydrodynamic mechanism in the transmission of pain producing stimuli through dentine. In: Anderson DJ, editor. Sensory mechanisms in dentine. Proceedings of a symposium held at the Royal Society of Medicine, London, September 24th, vol. 1962. Oxford: Pergamon Press; 1963. p. 73-9.

4. Addy M. Etiology and clinical implications of dentine hypersensitivity. Dent Clin N Am. 1990;34:503-14.

5. Canadian Advisory Board on Dentin Hypersensitivity. Consensus-based recommendations for the diagnosis and management of dentin hypersensitivity. J Can Dent Assoc (Tor). 2003;69:221-226.

6. Bekes K, Hirsch C. What is known about the influence of dentine hypersensitivity on oral health-related quality of life? Clin Oral Invest. 2013; 17(Suppl 1):S45-51.

7. Gibson B, Boiko OV, Baker S, Robinson PG, Barlow A, Player T, et al. The everyday impact of dentine sensitivity: personal and functional aspects. Soc Sci Dent. 2010;1:11-20.

8. Markowitz K. The original desensitizers: strontium and potassium salts. J Clin Dent. 2009;20:145-51.

9. Bae JH, Kim YK, Myung SK. Desensitizing toothpaste versus placebo for dentin hypersensitivity: a systematic review and meta-analysis. J Clin Periodontol. 2015;42:131-41.

10. West NX, Seong J, Davies M. Dentine hypersensitivity. In: Lussi A, Ganss C, editors. Erosive tooth wear. Monogr Oral Sci 2014;25:108-122.

11. Makin SA. Stannous fluoride toothpastes. Am J Dent. 2013;26(Spec No $A: 3 A-9 A$

12. Mason S, Hughes N, Sufi F, Bannon L, Maggio B, North M, et al. A comparative clinical study investigating the efficacy of a toothpaste containing $8 \%$ strontium acetate and 1040 ppm fluoride in a silica base and a control toothpaste containing $1450 \mathrm{ppm}$ fluoride in a silica base to provide immediate relief of dentin hypersensitivity. J Clin Dent. 2010;21(Spec Iss:42-8.

13. Hughes $N$, Mason S, Jeffery $P$, Welton $H$, Tobin M, O'Shea C, et al. A comparative clinical study investigating the efficacy of a test toothpaste containing 8\% strontium acetate and 1040 ppm sodium fluoride versus a marketed control toothpaste containing $8 \%$ arginine, calcium carbonate, and $1450 \mathrm{ppm}$ sodium monofluorophosphate in reducing dentinal hypersensitivity. J Clin Dent. 2010;21:9-55.

14. Gendreau L, Barlow AP, Mason SC. Overview of the clinical evidence for the use of NovaMin in providing relief from the pain of dentin hypersensitivity. J Clin Dent. 2011:22;90-5.

15. Pradeep AR, Sharma A. Comparison of clinical efficacy of a toothpaste containing calcium sodium phosphosilicate to a toothpaste containing potassium nitrate and to a placebo on dentinal hypersensitivity: a randomized clinical trial. J Periodontol. 2010;81:1167-73.

16. Nathoo S, Delgado E, Zhang YP, DeVizio W, Cummins D, et al. Comparing the efficacy in providing instant relief of dentin hypersensitivity of a new toothpaste containing $8.0 \%$ arginine, calcium carbonate, and 1450 ppm fluoride to a benchmark desensitizing toothpaste containing $2 \%$ potassium ion and $1450 \mathrm{ppm}$ fluoride and to a control toothpaste with 1450 ppm fluoride: a three-day clinical study in New Jersey, USA. J Clin Dent. 2009:20:123-30

17. Ayad F, Ayad N, Delgado E, Zhang YP, DeVizio W, et al. Comparing the efficacy in providing instant relief of dentin hypersensitivity of a new toothpaste containing 8.0\% arginine, calcium carbonate, and $1450 \mathrm{ppm}$ fluoride to a benchmark desensitizing toothpaste containing $2 \%$ potassium ion and $1450 \mathrm{ppm}$ fluoride, and to a control toothpaste with $1450 \mathrm{ppm}$ fluoride: a three-day clinical study in Mississauga. Canada J Clin Dent. 2009:20:115-22

18. Schiff T, Delgado E, Zhang YP, Cummins D, DeVizio W, hypersensitivity MLRD. Effective treatment with an in-office desensitizing paste containing $8 \%$ arginine and calcium carbonate. Am J Dent. 2009;22(Spec No A:3A-7A. 
19. Fu Y, Li X, Que K, Wang M, Hu D, Mateo LR, et al. Instant dentin hypersensitivity relief of a new desensitizing toothpaste containing 8.0\% arginine, a high cleaning calcium carbonate system and 1450 ppm fluoride: a 3-day clinical study in Chengdu, China. Am J Dent. 2010;23(Spec No:20A-7A.

20. West N, Newcombe RG, Hughes N, Mason S, Maggio B, Sufi F, et al. A 3-day randomized clinical study investigating the efficacy of two toothpastes, designed to occlude dentine tubules, for the treatment of dentine hypersensitivity. J Dent. 2013;41:187-94.

21. He T, Barker MI, Biesbrock AR, Sharma N. A randomized controlled clinical trial to assess the desensitizing effect of a stannous fluoride dentifrice. Am J Dent. 2014;27(2):106-10.

22. He T, Barker MI, Qaqish J, Sharma N. Fast onset sensitivity relief of a $0.454 \%$ stannous fluoride toothpaste. J Clin Dent. 2011;22:46-50.

23. He T, Cheng R, Biesbrock AP, Chang A, Sun L. Rapid desensitizing efficacy of a stannous-containing sodium fluoride toothpaste. J Clin Dent. 2011;22:40-5.

24. Parkinson C, Hughes N, Jeffery P, Jain R, Kennedy L, Qaqish J, et al. The efficacy of an experimental toothpaste containing $0.454 \% \mathrm{w} / \mathrm{w}$ stannous fluoride in providing relief from the pain of dentin hypersensitivity: an 8 week clinical study. Am J Dent. 2013;26:25A-31A.

25. Miller S, Truong T, Heu R, Stranick M, Bouchard D, Gaffar A. Recent advances in stannous fluoride technology: antibacterial efficacy and mechanism of action towards hypersensitivity. Int Dent J. 1994;44(Suppl 1):83-98.

26. Parkinson CR, Willson R. A comparative in vitro study investigating the occlusion and mineralization properties of commercial dentifrices in a four day dentine disc model. J Clin Dent. 2011;22:74-81.

27. Schiff T, He T, Sagel L, Baker R. Efficacy and safety of a novel stabilized stannous fluoride and sodium hexametaphosphate toothpaste for dentinal hypersensitivity. J Contemp Dent Pract. 2006;7:1-10.

28. Schiff T, Saletta L, Baker R, Winston Jl, He T. Desensitizing effect of a stabilized stannous fluoride/sodium hexametaphosphate toothpaste. Compendium Cont Ed Dent. 2005:26:35-40.

29. Parkinson CR, Hughes N, Hall NC, Whelton H, Gallob J, Mason S. Three randomized clinical trials to assess the short-term efficacy of anhydrous 0 . $454 \%$ w/w stannous fluoride toothpastes for the relief of dentin hypersensitivity. Am J Dent. 2016;29:25-32.

30. West NX, Seong J, Davies M. Management of dentine hypersensitivity: efficacy of professionally and self-administered agents. J Clin Periodontol. 2015;42(Suppl 16):S256-302.

31. The Procter \& Gamble Company. Oral care compositions which comprise stannous, potassium and monofluorophosphate. Patent W02011016984 A2. Publication date 10 Feb 201.

32. Glaxo group limited. Novel toothpaste composition. Patent WO2015028096 A1. Publication date 2015.

33. Earl JS, Langford RM. Physical and chemical characterization of the surface layers formed on dentin following treatment with an experimental anhydrous stannous fluoride toothpaste. Am J Dent. 2013;26:19A-24A

34. Blong MA, Volding $B$, Thrash WJ, Jones DL. Effects of a gel containing 0.4 percent stannous fluoride on dentinal hypersensitivity. Dent Hyg (Chic). 1985;59:489-92.

35. Miller JT, Shannon II, Kilgore W, Bookman JE. Use of a water-free stannous fluoride-containing gel in the control of dental hypersensitivity. J Periodontol. 1969:40:490-1.

36. Nehme M, Mason S, Hughes N, Targett D, Parkinson C, Tyson-Johnson $D$, et al. A randomized clinical study investigating the staining profile of an experimental stannous fluoride toothpaste. Am J Dent. 2013;26(Spec No A:32A-8A.

37. Burnett GR, Willson RJ, Lucas RA. In vitro studies investigating the dentin tubule-occlusion properties of an experimental anhydrous stannous fluoride toothpaste. Am J Dent 2013;26(Spec No A):10A-14A.

38. Burnett GR. The effect of an experimental anhydrous stannous fluoride toothpaste on the acid resistance of dentin smear layers. Am J Dent 2013b; 26(Spec No A):15A-18A.

39. Gallob J, Sufi F, Amini P, Siddiqi M, Mason S. A randomised exploratory clinical evaluation of dentifrices used as controls in dentinal hypersensitivity studies. J Dent. 2017:64:80-7.

40. Holland GR, Narhi MN, Addy M, Gangarosa L, Orchardson R. Guidelines for the design and conduct of clinical trials on dentine hypersensitivity. J Clin Periodontol. 1997;24:808-13.

41. Schiff T, Dotson M, Cohen S, De Vizio W, McCool J, Volpe A. Efficacy of a toothpaste containing potassium nitrate, soluble pyrophosphate, PVM/MA copolymer, and sodium fluoride on dentinal hypersensitivity: a twelve-week clinical study. J Clin Den. 1994;5(Spec No:87-92.

42. Polson AM, Caton JG, Yeaple RN, Zander HA. Histological determination of probe tip penetration into gingival sulcus of humans using an electronic pressure-sensitive probe. J Clin Periodontol. 1980;7:479-88.

43. Lobene RR, Weatherford T, Ross NM, Lamm RA, Menaker L. A modified gingival index for use in clinical trials. Clin Prev Dent. 1986;8:3-6.

44. Laster L, Laudenbach KW, Stoller NH. An evaluation of clinical tooth mobility measurements. J Periodontol. 1975:46:603-7.

45. American Dental Association. Acceptance program guidelines: products for the treatment of dentinal hypersensitivity. American Dental Association, Council on Scientific Affairs, 2012

46. Benedetti F. Placebo and the new physiology of the doctor-patient relationship. Physiol Rev. 2013;93:1207-46.

47. Addy M, West NX, Barlow A, Smith S. Dentine hypersensitivity: is there both stimulus and placebo responses in clinical trials? Int J Dent Hygiene. 2007;5:53-9.

48. West NX, Addy M, Jackson RJ, Ridge DB. Dentine hypersensitivity and the placebo response. A comparison of the effect of strontium acetate, potassium nitrate and fluoride toothpastes. J Clin Periodontol. 1997;24:209-15.

\section{Ready to submit your research? Choose BMC and benefit from:}

- fast, convenient online submission

- thorough peer review by experienced researchers in your field

- rapid publication on acceptance

- support for research data, including large and complex data types

- gold Open Access which fosters wider collaboration and increased citations

- maximum visibility for your research: over $100 \mathrm{M}$ website views per year

At BMC, research is always in progress.

Learn more biomedcentral.com/submissions 